\title{
Modelación con la ley constitutiva viscohipoplástica para estudiar la respuesta dinámica de suelos finos arcillosos
}

\begin{abstract}
Resúmen La microzonificación sísmica de Bogotá debe ser actualizada debido a que cuando se realizó su publicación en Agosto de 1997 existía mucha menos información que hoy. En muchos sitios es necesario realizar estudios locales de respuesta sísmica porque las características del subsuelo que se encuentra al realizar una perforación no coinciden con la descripción dada en la microzonificación. Estos estudios implican perforaciones, ensayos convencionales y dinámicos así como simulación de la propagación de ondas con modelos unidimensionales en la zona plana. La ley constitutiva viscohipoplástica es una alternativa para simular ensayos trixiales cíclicos, y otros, y permite estudiar la propagación de ondas en arcillas blandas con resultados mas realistas porque tiene en cuenta tanto el comportamiento viscoso como la no linealidad de estos suelos.
\end{abstract}

Abstract

Seismic zones in Bogota should be upgraded because of a wide variety of test and information which were not available when it was published in August 1997. In some places il's a must to carry on local seismic response studies because subsoil properties does not fit with available information form seismic zones. This studies involves new boreholes and conventional and dinamic test over soil samples and wave simulation with unidimensional models in flat areas. Viscohipoplastic constitutive model offers a new way to simulate ciclic triaxial, and also other tests and it is possible to study seismic wave propagation with more realistic results because it can handle viscous and non linear behavior of this kind of soils.

\author{
Alejandro Ulloa \\ aulloa@uniminuto.edu
}

\section{INTRODUCCIÓN \\ 1.1 Presentación del problema}

Existe preocupación por los efectos que podría tener un sismo de gran magnitud en Bogotá. Parte de la preocupación se debe al hecho que gran parte de la ciudad está mal construida, con material de baja calidad y sin cumplir normas legales ni técnicas. La otra cara de la moneda es que los precios de construcción se han incrementado notablemente en los últimos años, en particular lo concerniente al acero de refuerzo y una edificación puede costar entre un 20 y un $40 \%$ mas, dependiendo de la ubicación de la misma dentro de la ciudad en función de la zona de la microzonificación que le corresponda.

Se hacen estudios de respuesta local, a costos elevados y con dificultades metodológicas que pueden suplirse con la aplicación de la viscohipoplasticidad.

\subsection{Metodología}

En este trabajo se hicieron ensayos sobre muestras tomadas en cercanías al puente de la Calle 92 con Avenida NQS, en un lote donde fue construido un edificio de siete plantas. El subsuelo encontrado es una secuencia de arcillas y limos arcillosos blandos con humedades altas; sobre muestras seleccionadas se hicieron ensayos convencionales de consolidación, compresión inconfinada, límites y, adicionalmente, compresiones edométricas y triaxiales cíclicos al igual que un ensayo downhole. Un vez determinados los parámetros viscohipoplásticos, se hicieron simulaciones de los ensayos triaxiales cíclicos así como de las consolidaciones edométricas habiéndose logrado unos 
resultados satisfactorios lo cual permite sugerir que la viscohipoplasticidad constituye un método alterno para el desarrollo de estudios de respuesta local.

Ante la amenaza de un sismo no se puede hacer nada; ante nuestra vulnerabilidad frente al mismo sí, de tal manera que disminuya el riesgo. Con esta manera de pensar se han impulsado los estudios de respuesta local, que no son otra cosa que estudios orientados a estimar cómo se amplificaría una onda sísmica que viene propagándose por la roca basal en una cuenca como la Sabana de Bogotá y que llega a la superficie del terreno atravesando una secuencia de sedimentos lacustres blandos. Conocida la amplificación de la onda sísmica se puede generar el espectro de respuesta propio del sitio permitiendo hacer un diseño estructural con las características propias del sitio. Se ha encontrado que en no pocos casos los espectros obtenidos después de un estudio de respuesta local son menores (el factor de amplificación) a los especificados en la microzonificación con lo cual es posible disminuir de manera significativa el refuerzo de la estructura y las secciones lo cual conlleva menores costos de construcción, especialmente en edificios de mas de cinco pisos. De acuerdo con la normatividad vigente (Decreto 074 del 30 de enero de 2001) se pueden emplear espectros sísmicos de diseño diferentes a los de la microzonificación si se demuestra que fueron obtenidos utilizando mejor información proveniente de estudios detallados. Tal información consiste en realizar ensayos de velocidad de onda cortante por medio de métodos directos de campo tales como el crosshole, cono sísmico o equivalentes, ensayos de laboratorio para determinar el comportamiento del módulo dinámico de cortante y la capacidad histerética de amortiguamiento de muestras inalteradas representativas de todos los materiales del subsuelo. Se ordena el uso de ensayos de columna resonante, tor-sión cíclica, triaxial cíclico, corte simple cíclico u otros adecuados. Por otra parte, es necesario utilizar análisis dinámicos consistentes en evaluaciones en el tiempo utilizando modelos unidimensionales en el caso de la zona plana de Bogotá o bidimensionales en las zonas montañosas y también en las de pie de monte.

Finalmente, para la mencionada zona plana, se requiere hacer una perforación de por lo menos 50 metros de profundidad o bien hasta encontrar roca, si esta se haya a menor profundidad, de la cual se extraerán las muestras inalteradas para los ensayos.

\section{FUNDAMENTOS TEÓRICOS 2.1 Modelación y simulación}

Gran parte de la dificultad para resolver problemas en geotecnia era que, aunque se conocieran relaciones teóricas con fundamentos físicos entre las variables que intervienen en un determinado proceso, la solución matemática era muy compleja. Los avances en el desarrollo de los métodos numéricos y la disponibilidad de computadoras de gran capacidad han permitido en las últimas décadas el análisis de problemas geotécnicos que incluyen geometrías variables, condiciones de borde para resolver ecuaciones diferenciales y estudio del comportamiento mecánico de materiales cada vez más complejos.

Gracias a este desarrollo, un gran interés en el estudio y la comprensión del comportamiento de suelos y en la modelación matemática del mismo ha surgido en la comunidad geotécnica internacional, como lo prueban los diferentes talleres, simposios y conferencias referidos a este tema, en los últimos 30 años.

Hoy en día, la aplicación de métodos numéricos en la ingeniería. geotécnica se ve facilitada por un sinnúmero de programas de cómputo comerciales. A pesar de la gran libertad que pueden presentar estos programas, en lo que se refiere a la geometría de los modelos y a la elección de las condiciones de contorno, el número y la versatilidad de modelos constitutivos implementados es limitado.

Algunos programas permiten la inclusión de relaciones constitutivas utilizando interfaces, pero la implementación numérica de una nueva relación ley constitutiva es complicada y prácticamente limitada al campo académico. La disponibilidad y difusión de estos programas puede llevar a creer que cualquier usuario, inclusive aquellos sin demasiados conocimientos del comportamiento mecánico de suelos, pueden usarlos como "cajas negras" para resolver problemas geotécnicos en cualquier tipo de suelo pero ello no es así. El uso de estas herramientas de cómputo exige un profundo conocimiento del comportamiento real de los suelos para juzgar la calidad de los modelos constitutivos, de los cuales depende el realismo de las predicciones.

Existen diferentes modelos constitutivos del subsuelo creados para que, mediante relaciones matemáticas basadas en el estado crítico de los suelos, sea posible reproducir el comportamiento 
del suelo en cuanto a esfuerzos y deformaciones bajo diferentes condiciones de carga incluyendo las producidas por sismos o cargas de tipo dinámico en general.

Los modelos de simulación tienen una ventaja importante desde el punto de vista económico ya que, una vez conocidas algunas propiedades del subsuelo, obtenidas mediante ensayos convencionales, se puede reproducir su comportamiento y extrapolar resultados a otros estados de carga, sin necesidad de hacer ensayos adicionales.

En caso del estudio del subsuelo, la exploración y ensayos de laboratorio implican costos altos a causa, principalmente, de las perforaciones porque hoy en día se requieren ensayos que son costosos, tales como downhole, crosshole, triaxiales dinámicos y péndulo de torsión para obtener los módulos de Corte y Elástico, la relación de Poisson y el módulo de Bulk.

Por otra parte, el comportamiento del suelo bajo acciones dinámicas claramente no es ni elástico ni lineal y para acercarse a la respuesta real de un suelo no deben usarse los principios generales de la teoría elástica, la cual está basada en la hipótesis de una relación simple y lineal entre el esfuerzo y la deformación en cualquier material.

En la ciudad de Bogotá D.C., se han realizado varios ensayos sísmicos que permiten conocer las velocidades de propagación de las ondas compresionales $\left(V_{p}\right)$ y de corte $\left(V_{s}\right)$. Con base en esas velocidades, es posible calcular los llamados módulos dinámicos del suelo los cuales sirven de base para estudiar el comportamiento del suelo bajo acciones dinámicas tales como sismos y cargas repetidas o cíclicas.

Con base en la información recopilada sobre estos ensayos, se modela en este trabajo el comportamiento de las ondas bajo la hipótesis que el suelo tienen un comportamiento viscohipoplástico, teoría que es más cercana a la realidad del material, el cual, como todos sabemos, es elástico y lineal solamente en el rango de deformaciones muy pequeñas (en el orden de $10^{-5}$ ).

Una vez que se logran reproducir ensayos de diferentes tipos tales como consolidaciones y triaxiales, se puede ver el efecto que en el suelo arcilloso de Bogotá produce la propagación de ondas sísmicas para así acercarse a la realidad de los espectros de diseño presentados en el Código Colombiano Sismo-Resistente.
En otras palabras, basados en unas velocidades de propagación medidas, una vez se logre reproducirlas mediante simulación matemática y utilización de las leyes constitutivas viscohipoplásticas, se pueden formular espectros de diseño mas aproximados porque se podrá precisar mejor el efecto de amplificación, que producen los suelos blandos y arcillosos del depósito fluviolacustre de nuestra Sabana de Bogotá en la propagación de ondas sísmicas.

\subsection{Visco e hipoplasticidad}

La hipoplasticidad es un modelo constitutivo inelástico e incrementalmente no-lineal, el cual no requiere de una superficie de fluencia ni de la descomposición de la tasa de deformación en una porción elástica y otra plástica. Tiene la habilidad de describir comportamientos disipadores, flujos plásticos y efectos no-lineales dentro de la superficie de fluencia, con una simple ecuación tensorial.

El concepto de hipoplasticidad fue introducido por D. Kolymbas (1977), aunque modelos similares habían sido tratados de forma independiente por diferentes investigadores. Kolymbas sugirió el nombre "hipoplásticos" para los modelos con rigidez tangencial que fueran función continua de la tasa de de-formación, siendo el prefijo "hipo" justificable para aquellos modelos menos restrictivos que la elastoplasticidad.

La hipoplasticidad reconoce que las deformaciones inelásticas pueden presentarse desde el inicio del proceso de carga, sin distinguir a priori entre las deformaciones plásticas y las elásticas. La característica sobresaliente de la hipoplasticidad es que emplea una única ecuación que se mantiene para carga y descarga. Esta distinción entre carga y descarga es automáticamente consumada por la ecuación misma. La familia de investigadores, encuentra que la hipoplasticidad es fácil de implementar en algoritmos numéricos y también fácil para ser comprendida.

La ecuación constitutiva hipoplástica expresa el incremento del esfuerzo como una función dada por el incremento de la deformación y del esfuerzo y la relación actual de vacíos. En lugar de incrementos de esfuerzo y deformación, se habla de tasas de esfuerzo y deformación. Se puede concebir la tasa de esfuerzo como un incremento obtenido en una unidad de tiempo. Dado que el esfuerzo y la deformación son cantidades tensoriales, la ecuación hipoplástica es una ecuación tensorial. 
Como cualquier ecuación constitutiva hay varias versiones de ecuaciones hipoplásticas, unas recientes y unas más avanzadas. La ecuación original hipoplástica fue publicada por D. Kolymbas, en 1977, y luego versiones mejoradas fueron introducidas por algunos autores.

En los años noventa, fueron desarrolladas numerosos trabajos en este campo por grupos de investigadores como el de la Techinical University of Karlsruhe, entre los cuales se encuentran los profesores A. Niemunis, G. Gudehus y D. Kolymbas. Los resultados por ellos alcanzados, han llevado a continuar con las investigaciones hacia diversas aplicaciones.

La hipoplasticidad es un modelo que no ha sido derivado de leyes fundamentales o físicas, sino que se basa en datos experimentales, por lo que se considera de tipo fenomenológico. Adicionalmente, describe el comportamiento del suelo en términos de macro variables, como el esfuerzo o la relación de vacíos, considerándolo como un medio continuo para un acercamiento macromecánico que no describe el movimiento individual de las partículas.

\subsection{Fundamentos físico-matemáticos}

La ley constitutiva viscohipoplástica (Niemunis, 2003) tiene la siguiente forma:

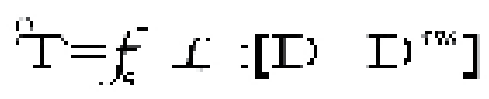

en donde $\mathbf{D}$ representa la tasa de deformación presentada en dos partes una elástica y una viscosa.

El término $f_{b}$ es el factor de barotropía y $\mathcal{L}$ es un tensor de cuarto orden. La parte viscosa se representa por una expresión del tipo:

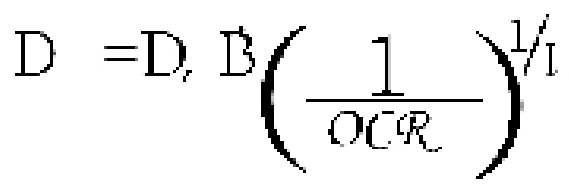

(2)

En la expresión (2), Dr es la deformación de referencia, B es la dirección del creep y el término IV corresponde al índice de viscosidad.

Estas ecuaciones fueron adecuadas por Niemunis en 2003 para describir el comportamiento viscoso de los suelos arcillosos, para poder obtener parámetros que permitan modelar a partir de ensayos triaxiales y edométricos y porque fue necesario mejorar el modelo para que representara adecuadamente los triaxales en pequeños ciclos de carga.

De allí nació el concepto de deformación intergranular, el cual considera la rigidez momentánea causada por el cambio de dirección de un movimiento cíclico. De esta manera la ecuación (1) toma la siguiente forma:

$$
\left.\left.\Gamma=f_{c} M([1]-]\right)^{\mathrm{m}}\right]
$$

(3)

En la expresión (3) aparece el término $\mathrm{M}$ el cual es un tensor de cuarto orden dependiente de la historia reciente de deformaciones del suelo.

Este tensor se obtiene a partir de las variables $\mathrm{mR}$, y mT, las cuales a su vez son función del sentido de deformación hacia un lado o en sentido inverso ( reversa).

La ecuación para la ley constitutiva hipoplástica requiere de ocho parámetros del material; en la tabla 1 se indican los nombres de los mismos y el ensayo de donde se obtienen ( $\mathrm{E}=$ test edométri$c o, T=$ prueba triaxial):

\begin{tabular}{|c|c|c|c|c|c|c|c|}
\hline $\mathrm{e}_{100}$ & $\lambda$ & $\mathcal{K}$ & $\beta$ & $\mathrm{I}_{\mathrm{v}}$ & $\mathrm{D}_{\mathrm{r}}$ & $\varphi_{\mathrm{c}}$ & $\mathrm{OCR}$ \\
\hline $\mathrm{E}$ & $\mathrm{E}$ & $\mathrm{E}$ & $\mathrm{T}$ & $\mathrm{E}$ & $\mathrm{E}$ & $\mathrm{T}$ & $\mathrm{E}$ \\
\hline
\end{tabular}

Tabla 1. Parámetros hipoplásticos

La relación constitutiva viscohipoplástica se basa en la teoría de la hipoplasticidad y describe el comportamiento viscoso de suelos blandos sometidos a carga predominantemente monotónica en condiciones de drenada y no drenada.

Los parámetros del modelo constitutivo viscohipoplástico son adicionalmente $m_{R^{\prime}} m_{T^{\prime}} R_{\text {max }^{\prime}} \beta x, x$ la velocidad de deformación. I se puede determinar con ensayos de compresión edométrica. Alternativamente, $I_{v}$ puede ser determinado por ensayos de compresión no drenada con cambios bruscos (saltos) de la tasa de deformación.

El ángulo de fricción interna crítico corresponde aproximadamente al estado residual, que se obtiene en ensayos de comprensión triaxial drenado o no drenado de muestras normalmente consolidadas y a diferencia de las teorías elastoplásticas, los parámetros del modelo viscohipoplásti- 
co son independientes del estado del material y pueden ser determinadas en el laboratorio usando muestras remodeladas.

\subsection{Simulaciones de consolidaciones y triaxiales cíclicos}

Se escogieron muestras de arcillas blandas a profundidades entre 10 y 12 metros tomadas en tubos Shelby, ubicadas en la calle 93 bis con avenida NQS de la ciudad de Bogotá. El sitio presenta el clásico problema que no es muy claro si es de la zona 3 o de la zona 4; las muestras y ensayos realizados lo clasifican sin lugar a dudas en la zona 4, lacustre B caracterizada por arcillas blandas de gran espesor.

La perforación se llevó hasta $40 \mathrm{~m}$. de profundidad y se tomaron gran cantidad de muestras de una secuencia de suelos arcillo limosos y limo arcillosos blandos. Además, de estas muestras se consultaron diferentes estudios de suelos de los alrededores con suelos de características similares, realizados por diferentes empresas.

Típicamente los suelos tienen humedades naturales de 130 a $145 \%$, límite líquido de 160 a 173\%, límite plástico del orden de 56\%, índice de plasticidad cercano a $117 \%$, índice de liquidez aproximadamente de $76 \%$, se clasifica claramente como un $\mathrm{CH}$, y su peso unitario es de $12.7 \mathrm{kN} / \mathrm{m} 3$.

En estas muestras se hicieron ensayos de compresión inconfinada, edométricos, consolidaciones y triaxiales cíclicos. Se prestó especial atención a estos últimos ya que son representativos del comportamiento dinámico del suelo; de hecho se realizaron ensayos en tres universidades para comparar los resultados y tener así mejores elementos de juicio a la hora de hacer la simulaciones.

Para las modelaciones con viscohipoplasticidad se encontró que algunos de los parámetros varían en tantos que otros permanecen prácticamente constantes.

En efecto, las mediciones efectuadas indican lo siguiente:

El parámetro $\lambda$, varía normalmente entre 0.21 y 0.29; no obstante parecen mas comunes valores del orden de 0.22 a 0.25 .

El ángulo de fricción $\varphi_{c}$ es comúnmente $18^{\circ}$ para las arcillas estudiadas en Bogotá pero también existen valores del orden de $15^{\circ}$. Nótese que la existencia del ángulo de fricción es atribuible a la presencia de limo ya que la arcilla sin limo es considerada con ángulo de fricción nulo.

El término $\mathrm{k}$, presenta mayores variaciones pues se encontraron mediciones entre 0.021 y 0.047 , es decir, la relación entre los menores valores y los mayores es cercana a 2.

El índice de viscosidad IV se ubica entre 0.050 y 0.065, habiéndose encontrado los valores mas comunes casi siempre 0.055 y 0.060 .

El valor $\beta$ se usa siempre como 0.95, sin variaciones.

La relación de sobre consolidación OCR, varía entre 1.2 y 1.8 para las muestra estudiadas.

La relación de vacíos e100 se encontró que se movía entre 2.2 y 3.42 , rango amplio.

Los parámetros relacionados con la deformación intergranular indispensables para los ensayos cíclicos, mostraron lo siguiente:

El término $m_{R}$ siempre fue menor que $m_{T^{\prime}}$ el primero se sitúa alrededor de 2 a 3 y el segundo cerca-no a 5 o 6.

El parámetro X está entre 0.9 y 2 aproximadamente, aunque se tiene información de valores mas altos y mas bajos usados en otras simulaciones.

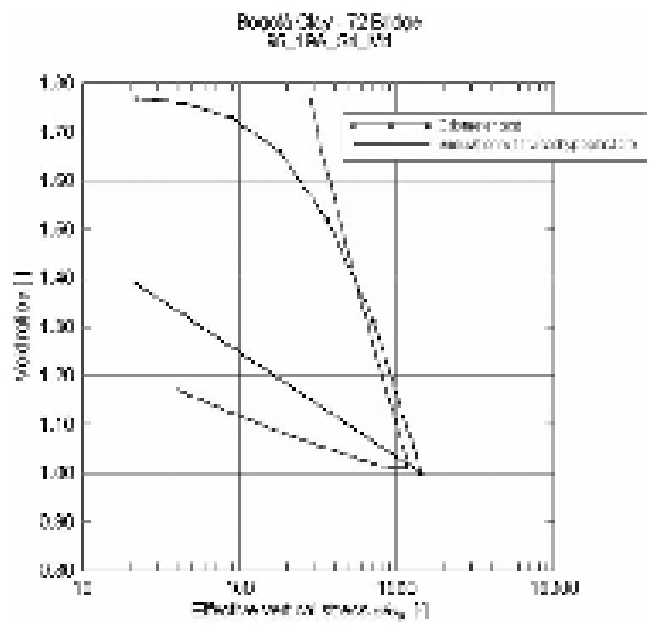

Figura 1. Simulación viscohipoplástica sobre un ensayo de consolidación rápida convencional

Nótese que la simulación reproduce aproximadamente bien el ensayo, pero se nota una diferencia entre el modelo y el ensayo de laboratorio en la zona de descarga. Esto es debido a que en el ensayo de laboratorio no existen sino dos 
puntos para la descarga: el primero es el que corresponde a la finalización de la carga y el segundo la finalización de la descarga sin puntos intermedios lo cual obliga a unirlos con una línea recta. Es probable que la simulación sea correcta pero no se puedan comparar en este tramo por lo anotado.

En la simulación hecha en este ensayo se trabajó sobre lo que comúnmente se llama ensayo de "consolidación rápida". El proceso que se hace es no dejar que la muestra llegue a consolidarse sino dejarla un tiempo fijo descargar y aumentar la carga, y así sucesivamente; generalmente, por dos o tres ciclos como máximo aunque se hace con un solo ciclo de carga y descarga lo cual dificulta la obtención de los parámetros viscohipoplásticos.

La figura 2 muestra una simulación hecha sobre una muestra tomada adecuadamente y ensayada para determinar parámetros viscohipolásticos en la calle 93bis\# 20-33. En esta muestra se hizo un ensayo que comercialmente se llama "consolidación larga" el cual permite mejorar la aproximación de la simulación, tal como puede verse en la línea punteada la cual está claramente muy cerca del ensayo realizado. Se podría concluir que a pesar de que los ensayos no se hagan con todos los cuidados la ley constitutiva viscohipoplástica da la tendencia de la curva tal como se ve en la figura 1 y que si se hacen cuidadosamente los ensayos se obtiene buen ajuste, como en la figura 2.

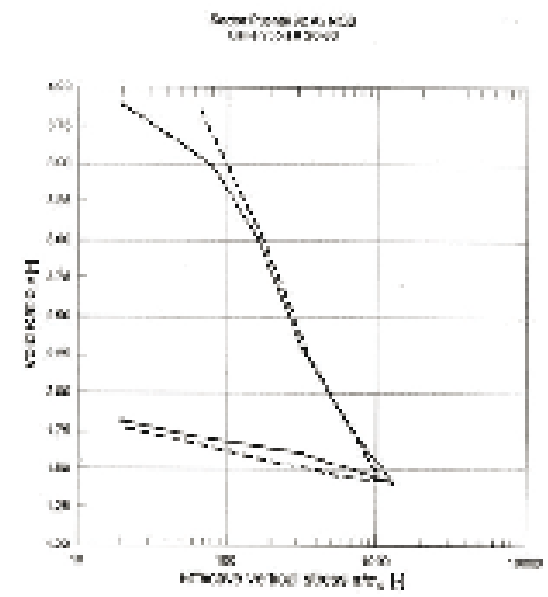

Figura 2. Ensayo de consolidación largo, simulado con viscohipoplasticidad

En cuanto a lo que se refiere a los ensayos traxiales cíclicos se tomaron muestras prácticamente de la misma profundidad y se ensayaron en dos laboratorios diferentes, habiéndose encontrado que también es factible simularlos con viscohipoplasticidad.
La figura 3 muestra un ensayo realizado, en EAFIT en Medellín, cuidadosamente controlado y puede observarse que la simulación y la medición coinciden bien.

\section{SMULACION TRIAX AL CICLIEOEAFIT}

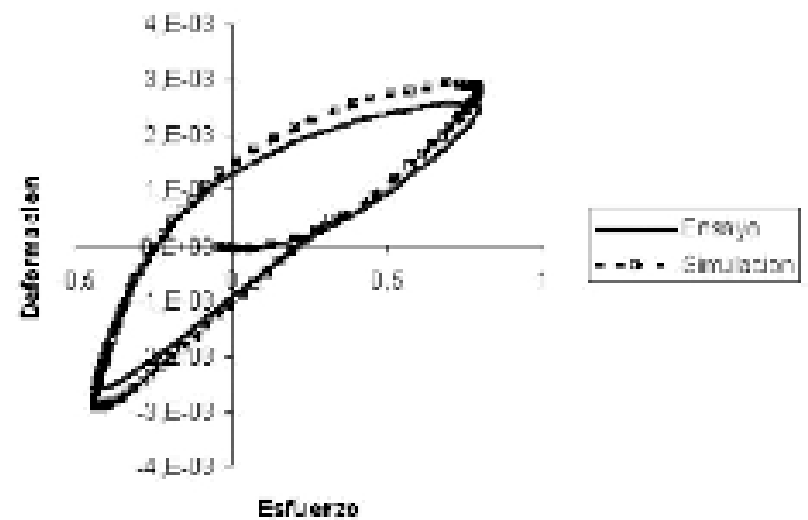

Figura 3. Simulación ensayo triaxial cíclico, EAFIT

La figura 4 muestra el ensayo triaxial cíclico realizado en la Universidad de Los Andes y en él también se ve la coincidencia entre lo experimental y la simulación.

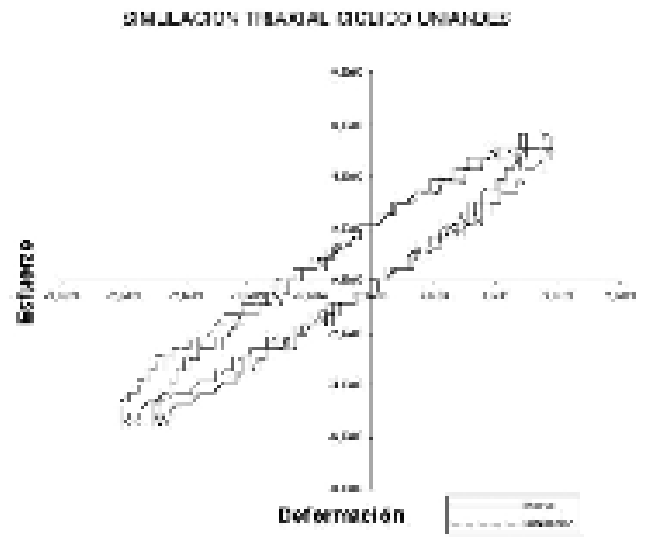

Figura 4. Simulación triaxial cíclico, realizada en Uniandes

\section{Conclusiones}

La modelación o simulación con viscohipoplasticidad reproduce con bastante precisión ensayos de consolidación y traxiales cíclicos inclusive puede reproducir ensayos comerciales o convencionales realizados sin todos los cuidados tales como las llamadas "consolidaciones rápidas".

De esta manera, se puede afirmar que se pueden obtener las curvas de degradación del módulo de corte $G$, a partir de las simulaciones, y consecuentemente se puede simular la propagación 
de ondas sísmicas siendo esta la base para la generación de espectros de diseño.

Los módulos de corte $G$ obtenidos a partir de los ensayos simulados fueron comparados con ensayos downhole y se encontró que coinciden en cuanto al valor Gmax que es el que se obtiene de un downhole.

Las metodologías empleadas para los ensayos triaxiales siguieron la norma ASTM 03999-91 en los laboratorios empleados y se observa que la simulación es capaz de reproducir los ensayos.

\section{Bibliografía}

Astm (1996) Standard Test Methods for the de-termination of the Modulus and Camping Properties of Soils Using the Cyclic Triaxial Apparatus.

Crice, Douglas. (1995). Shear Waves Techniques and Systems, Geometrics Inc.

Cudmani, R (2003). Aspectos fundamentales del comportamiento de suelos desde una perspectiva histórica y su modelación a través de las relaciones constitutivas hipoplásticas. Instituto de Mecánica de Suelos y Rocas, Universidad de Kalsruhe, Alemania

Decreto 074 de Enero 30 de 2001, Por el cual se complementa y se modifica el Código de Construcción de Bogotá Distrito Capital, Alcaldía Mayor. Ingeominas, Universidad de Los Andes, Upes (1997). Microzonificación sísmica de Santafé de Bogotá.

Grupo Cecata Universidad Javeriana (2002).Caracterización de los parámetros dinámicos de las arcillas blandas de Bogotá sobre el corredor vial de la Avenida Boyacá.

Lizcano, A., Reyes, D.K., Grandas, C. (2004) Site Response Análisis Using a Visco-Hypoplastic Model, Universidad de Los Andes.

Niemunis, A (2003). Extended hypoplastic models for soils. Des Institutes fur Grundbau und Boden-mechanik der ruhr-universitat Bochum Tatsuoka F., Shibuya S., Kuwano R. (2001) Advanced Laboratory Stress-Strain Testing of Geomaterials, Tokio. 